\title{
Universiteit
}

Leiden

The Netherlands

\section{The effects of cortisol administration on approach-avoidance behavior: An event-related potential study.}

Peer, J.M. van; Roelofs, K.; Rotteveel, M.; Dijk, J.G. van; Spinhoven, P.; Ridderinkhof, K.R.

\section{Citation}

Peer, J. M. van, Roelofs, K., Rotteveel, M., Dijk, J. G. van, Spinhoven, P., \& Ridderinkhof, K. R. (2007). The effects of cortisol administration on approach-avoidance behavior: An eventrelated potential study. Biological Psychology, 76(3), 135-146. Retrieved from https://hdl.handle.net/1887/13164

Version: $\quad$ Not Applicable (or Unknown)

License: $\quad$ Leiden University Non-exclusive license

Downloaded from: https://hdl.handle.net/1887/13164

Note: To cite this publication please use the final published version (if applicable). 


\title{
The effects of cortisol administration on approach-avoidance behavior: An event-related potential study
}

\author{
Jacobien M. van Peer ${ }^{\mathrm{a}, *}$, Karin Roelofs ${ }^{\mathrm{a}}$, Mark Rotteveel ${ }^{\mathrm{b}}$, J. Gert van Dijk ${ }^{\mathrm{c}}$, \\ Philip Spinhoven ${ }^{\mathrm{a}, \mathrm{d}}$, K. Richard Ridderinkhof ${ }^{\mathrm{e}, \mathrm{f}}$ \\ a Department of Clinical, Health and Neuropsychology, Leiden University, PO Box 9555, 2300 RB Leiden, The Netherlands \\ ${ }^{\mathrm{b}}$ Department of Social Psychology, University of Amsterdam, Roetersstraat 15, 1018 WB Amsterdam, The Netherlands \\ ${ }^{\mathrm{c}}$ Departments of Neurology and Clinical Neurophysiology, Leiden University Medical Centre, PO Box 9600, 2300 RC Leiden, The Netherlands \\ ${ }^{\mathrm{d}}$ Department of Psychiatry, Leiden University, PO Box 9600, 2300 RC Leiden, The Netherlands \\ ${ }^{\mathrm{e}}$ Department of Psychology, Leiden University, PO Box 9555, 2300 RB Leiden, The Netherlands \\ ${ }^{\mathrm{f}}$ Amsterdam Center for the Study of Adaptive Control in Brain and Behavior, Department of Psychology, \\ University of Amsterdam, Roetersstraat 15, 1018 WB Amsterdam, The Netherlands
}

Received 19 January 2007; accepted 14 July 2007

Available online 20 July 2007

\begin{abstract}
We investigated the effects of cortisol administration $(50 \mathrm{mg})$ on approach and avoidance tendencies in low and high trait avoidant healthy young men. Event-related brain potentials (ERPs) were measured during a reaction time task, in which participants evaluated the emotional expression of photographs of happy and angry faces by making an approaching (flexion) or avoiding (extension) arm movement. The task consisted of an affect-congruent (approach happy faces and avoid angry faces) and an affect-incongruent (reversed instruction) condition. Behavioral and ERP analyses showed that cortisol enhanced congruency effects for angry faces in highly avoidant individuals only. The ERP effects involved an increase of both early (P150) and late (P3) positive amplitudes, indicative of increased processing of the angry faces in high avoidant subjects after cortisol administration. Together, these results suggest a context-specific effect of cortisol on processing of, and adaptive responses to, motivationally significant threat stimuli, particularly in participants highly sensitive to threat signals.
\end{abstract}

(C) 2007 Elsevier B.V. All rights reserved.

Keywords: Cortisol; Approach-avoidance; Facial expression; Action tendencies; Event-related potentials; Behavioral Inhibition Scale

Activity of the hypothalamus-pituitary-adrenal (HPA) axis is important in the regulation of adaptive stress responses such as the generation of active avoidance reactions (see Sapolsky et al., 2000). Stress leads to activation of the HPA system, resulting in the release of endogenous glucocorticoids such as cortisol. Particularly when measured in social situations, elevated cortisol levels have been found to be related to the manifestation of social submissiveness and avoidance behavior (Sapolsky, 1990). Despite the extensive literature on the relation between HPA-axis activity and avoidance behavior in animals, little is known about the role of cortisol in the generation of human avoidance behavior. In this study, we examined the effect of cortisol administration on avoidance reactions to threatening social stimuli (angry faces) in human

\footnotetext{
* Corresponding author. Tel.: +31 71 5273835; fax: +31 715274678 .

E-mail address: jpeer@fsw.leidenuniv.nl (J.M. van Peer).
}

participants. In addition, to gain more insight in the brain processes underlying these reactions, we measured eventrelated brain potentials (ERPs) during performance of an approach-avoidance task, specifically focusing on positive components related to emotional face processing.

The generation of active avoidance responses depends on a motivational network that involves various brain regions (see LeDoux, 2002; Rolls, 2000). When threat stimuli are processed by the amygdala, direct autonomic responses and primary motor reactions such as freezing are activated via connections to the brainstem. Moreover, motivational systems are activated that guide instrumental responses based on past learning or instantaneous decisions. The hippocampus and prefrontal cortex (PFC) play an important role in these motivational systems. The PFC is thought to integrate information on arousal (from brainstem centers) with context-relevant information (from the hippocampus) and with temporary contents of working memory (from PFC areas) in controlling motor 
responses (via connections with the motor cortex). The anterior cingulate (ACC) and orbitofrontal (OFC) regions of the PFC in particular are involved in these motivational systems, which enable approach and avoidance reactions to emotional stimuli (see LeDoux, 2002; Roelofs et al., in preparation; Rolls, 2000).

Rolls (2000) stressed the importance of processing of facial expressions by these motivational systems. Emotion has a communicative function, and faces constitute important signals of threat or appeasement in the social environment. In a series of lesion studies, Hornak et al. (2003) showed that in human participants both the OFC and the ACC are involved in emotion processing, including the identification of facial expression, social behavior, and subjective emotional state.

Angry facial expressions are commonly used as social threat stimuli in human research on threat processing. Neuroimaging studies have shown that viewing angry faces activates large parts of the above-mentioned motivational network, with the ACC, OFC, and amygdala in particular (for an overview see Adolphs, 2002; McClure et al., 2004; Strauss et al., 2005). In addition, transcranial magnetic stimulation of the medial PFC/ ACC has been found to disrupt the processing of angry facial expressions (Harmer et al., 2001). Adolphs (2002) argued that whereas activation of the amygdala appears to depend on relatively passive or implicit processing of the emotion (such as in passive viewing paradigms), prefrontal regions may be activated more when participants are engaged in a cognitive task requiring explicit identification of the emotion, which in turn may inhibit the amygdala's activation.

ERP studies have also indicated that prefrontal motivational networks are involved in the processing of facial expressions. An enhanced positivity in response to emotional relative to neutral faces has been found over prefrontal areas as early as $120 \mathrm{~ms}$ after stimulus presentation (Eimer and Holmes, 2002) or between 160 and $215 \mathrm{~ms}$ (Eimer et al., 2003). This suggests that cortical circuits involved in the detection of emotionally significant events can be triggered rapidly by emotional facial expressions (Eimer et al., 2003; Pizzagalli et al., 1999; Sato et al., 2001). In addition, a more broadly distributed positivity (over parietal as well as frontal and central areas) has been observed beyond $300 \mathrm{~ms}$ (Eimer et al., 2003). In particular faces signaling threat (i.e. fearful or angry faces as opposed to happy or neutral faces) have been found to show these enhanced amplitudes in both early (e.g. 50-250 ms: Ashley et al., 2004; Bar-Haim et al., 2005; Schupp et al., 2004; Williams et al., 2006) and late positive components (300-500 ms: Schupp et al., 2004; Williams et al., 2006). Interestingly, recent studies reported the ERP effects of emotional expressions to be attention dependent (Eimer et al., 2003; Krolak-Salmon et al., 2001), suggesting they may reflect a greater allocation of attention to motivationally relevant input (Cuthbert et al., 2000).

In sum, a frontolimbic motivational network is involved in the processing of social threat stimuli and the generation of avoidance behavior. In the next section we explore how the stress hormone cortisol, which is thought to be important in the generation of adaptive stress responses (e.g. Sapolsky et al., 2000), may affect this network and, consequently, approach and avoidance behavior. It is well established that not only the hippocampus but also the PFC is a target structure for cortisol (e.g. Meaney and Aitken, 1985; Radley et al., 2004). Exogenously administered cortisol has been shown to affect prefrontal functions, such as working memory, in humans (for a review see Wolf, 2003). In addition, there is increasing evidence from animal studies that PFC mediated avoidance behavior and fearful temperament are positively correlated with high levels of cortisol (see e.g. Kalin et al., 1998a,b, 2000). De Kloet et al. (1999) emphasized that glucocorticoids influence informationprocessing systems conditionally, so that specific internal and external stimuli are more likely to elicit responses in the appropriate context. In this way, information processing is biased towards adaptive behavior that is most relevant to the situation.

Human studies on the relation between cortisol, the processing of social threat stimuli and avoidance behavior are scarce, but a recent study by Putman et al. (2007) suggested that acute $(25 \mathrm{mg})$ cortisol administration enhanced preferential processing of angry faces in healthy young men. The results of this study showed a significant increase in memory bias for angry faces (i.e. enhanced spatial working memory performance compared to neutral faces) after cortisol administration compared to placebo. No such memory bias was found for happy faces. In addition, a study by Van Honk et al. (1998) in which angry and neutral faces were presented in a Stroop paradigm indicated that increased basal cortisol levels were associated with faster responses to angry faces, which was interpreted as reflecting (adaptive) avoidance. However, no studies so far have addressed the effects of cortisol administration on overt avoidance behavior.

A systematic and objective method to study human avoidance behavior was provided by Solarz (1960) and Chen and Bargh (1999), consisting of a reaction time task in which individuals evaluate the emotional valence of positive and negative word stimuli by making arm movements (arm flexion or extension) that are either congruent or incongruent with their intuitive action tendencies. Rotteveel and Phaf (2004) extended this paradigm to the nonverbal domain, using pictures of happy and angry faces (the approach-avoidance (AA) task). Affectcongruent movements involve arm flexion (approach) in response to a positive stimulus (happy face) and arm extension (avoidance) in response to a negative stimulus (angry face). Affect-incongruent movements involve reversed mapping instructions (from stimulus valence to arm movement) that conflict with participants' intuitive action tendencies (i.e. to approach positive and avoid negative stimuli). With this paradigm a congruency effect is typically found, indicating faster responses for affect-congruent arm movements compared to affect-incongruent arm movements (see also Chen and Bargh, 1999; Markman and Brendl, 2005; Solarz, 1960).

Using this AA task, Roelofs et al. (2005) found an effect of stress-induced cortisol responses on the congruency effects. Participants with relatively high stress-induced cortisol responses (high $\mathrm{CR}$ ) showed increased AA congruency effects when tested in baseline conditions, but no significant congruency effects during stress. In contrast, for low CR participants the congruency effects were only significant during and not before stress. Thus, the results of this study showed a significant 
interaction of cortisol response and stress on approachavoidance tendencies as measured by the AA task. However, the effects of high stress-induced cortisol levels could not be disentangled from the influence of individual differences in stress responsiveness or the effect of the social stress context. Therefore, the present study aimed to further investigate the effects of high cortisol levels on approach-avoidance tendencies, by studying the effects of cortisol administration on behavioral responses (particularly threat avoidance) in the AA task.

In addition, to investigate the effects of individual differences in threat sensitivity on behavioral responses to the threat signaling angry faces in the AA task, we compared participants with high scores to participants with low scores on a self-report measure of threat sensitivity (the Behavioral Inhibition Scale (BIS): Carver and White, 1994). Individuals with high scores on this scale (high BIS participants) can be characterized as anxiety prone, and tend to avoid threat (Carver and White, 1994). Compared to low BIS participants, we expected high BIS participants to be particularly responsive to social threat cues and to show relatively increased avoidance tendencies to the angry faces.

To test the effects of cortisol on these avoidance reactions, we administered the AA task to both participant groups after placebo and cortisol (hydrocortisone) administration. Because high cortisol levels have been associated with context-relevant adaptive responses (De Kloet et al., 1999; Sapolsky et al., 2000), biased processing of angry faces (Putman et al., 2007), and increased avoidance responses to threat (Buss et al., 2003; Kalin et al., 1998a,b, 2000; Van Honk et al., 1998), we expected cortisol administration to result in relatively increased avoidance reactions to angry faces on the AA task. Furthermore, we hypothesized that this effect would be especially strong for the high BIS subjects, given their increased sensitivity to these social threat cues. Such increased threat avoidance in the AA task can be either manifested by an increase in the effect of arm movement (faster avoidance than approach movements) for angry faces, or an increase in the effect of emotional expression for avoidance reactions (faster avoidance of angry than happy faces).

The second purpose of this study was to investigate brain processes associated with these effects using ERPs, with specific focus on components involved in emotional face processing and action monitoring. ERP components of particular interest were the previously mentioned positive waves that have been found over the prefrontal cortex between 120 and $250 \mathrm{~ms}$ poststimulus, and the more broadly distributed positive wave observed beyond $300 \mathrm{~ms}$ (e.g. Eimer et al., 2003; Schupp et al., 2004). In line with our behavioral expectations, we expected cortisol administration to result in increased amplitudes of these components especially in the high avoidant (high BIS) participants during avoidant reactions to angry faces. ${ }^{1}$

\footnotetext{
${ }^{1}$ In contrast, the face-specific N170 component, which can be recorded over posterior temporal areas, has been found to be relatively insensitive to emotion processing and is predominantly associated with structural encoding of faces (see e.g. Ashley et al., 2004). We therefore had no predictions regarding this component with relevance to approach and avoidance behavior.
}

A final component of interest was the N2, a frontocentral negative wave arising 200-350 ms post-stimulus. The N2 has been found to be increased in high conflict conditions, when incompatible response tendencies are simultaneously activated, and is suggested to reflect action monitoring (e.g. Van Veen and Carter, 2002), a function served by the medial prefrontal cortex (Ridderinkhof et al., 2004). In the AA task such response conflict may be elicited by affect-incongruent trials where the executed response is hypothesized to be in conflict with the participants' intuitive response tendency (i.e. to approach happy and avoid angry faces) (see Chen and Bargh, 1999; Rotteveel and Phaf, 2004). This study allows exploring whether the AA task indeed elicits significant N2 effects and whether cortisol administration may affect action monitoring during the generation of approach-avoidance responses.

To summarize our major predictions, we expected that cortisol administration would result in a facilitation of threat avoidance in high BIS participants. In addition, these behavioral effects were expected to be accompanied by increased amplitudes of ERP components involved in emotional face processing (in particular social threat). Finally, we explored whether cortisol administration would also increase action monitoring in high BIS participants.

\section{Methods}

\subsection{Participants}

Forty male students recruited from the University of Leiden participated in the experiment for financial (i.e. $€ 40$ ) or course credit. To create two extreme groups that differed in threat sensitivity, we selected a priori 20 students with low scores $(\leq 16)$ and 20 students with high scores $(\geq 21)$ on the Behavioral Inhibition Scale (BIS: Carver and White, 1994, see trait measures). Cutoff scores for these groups were based on the lower third and the upper third of the distribution of BIS scores (range 9-28, $M=18.5$, S.D. $=3.6$ ) in a sample of 153 male students.

Participants in this study were screened with the General Health Questionnaire (GHQ-12 ( $M=1.45$, S.D. = 1.69): Goldberg, 1978; Dutch version: Koeter and Ormel, 1991) and a biographic questionnaire to exclude any psychiatric disorder, clinical significant medical disease, past head injury with loss of consciousness $>5 \mathrm{~min}$, and use of medication. Inclusion criteria were right-handedness, normal or corrected-to-normal vision, age 18-30, and bodyweight $60-85 \mathrm{~kg}$. Participants were instructed to minimize physical exercise, not to take large meals, chocolate or caffeine during the morning preceding the experiment, and not to eat, drink low $\mathrm{pH}$ drinks or smoke cigarettes in the hour before the start of the experiment, because these variables can affect saliva cortisol measurements. All participants provided written informed consent prior to participation in the study, which was approved by the Medical Ethical Committee of the Leiden University Medical Center.

\subsection{Materials and procedure}

All participants were tested in a hydrocortisone $(50 \mathrm{mg})$ and a placebo condition in a double-blind, within-subject crossover design. The order of cortisol or placebo administration (i.e. a capsule) was random and balanced within the high and low BIS groups. The two experimental sessions were 1 week apart. On the days of testing, participants arrived at the laboratory at either 12.15 or 2.15 p.m. After a short introduction, drug administration followed at 12.30 or 2.30 p.m., respectively. After ingestion of the capsule, a resting period of $1 \mathrm{~h}$ followed to allow for the cortisol to take effect. During this period, participants completed questionnaires and practiced with the response device for the approach-avoidance task, after which the electrodes for the electrophysiological measurements were placed. Subsequently, the experiment started with a 

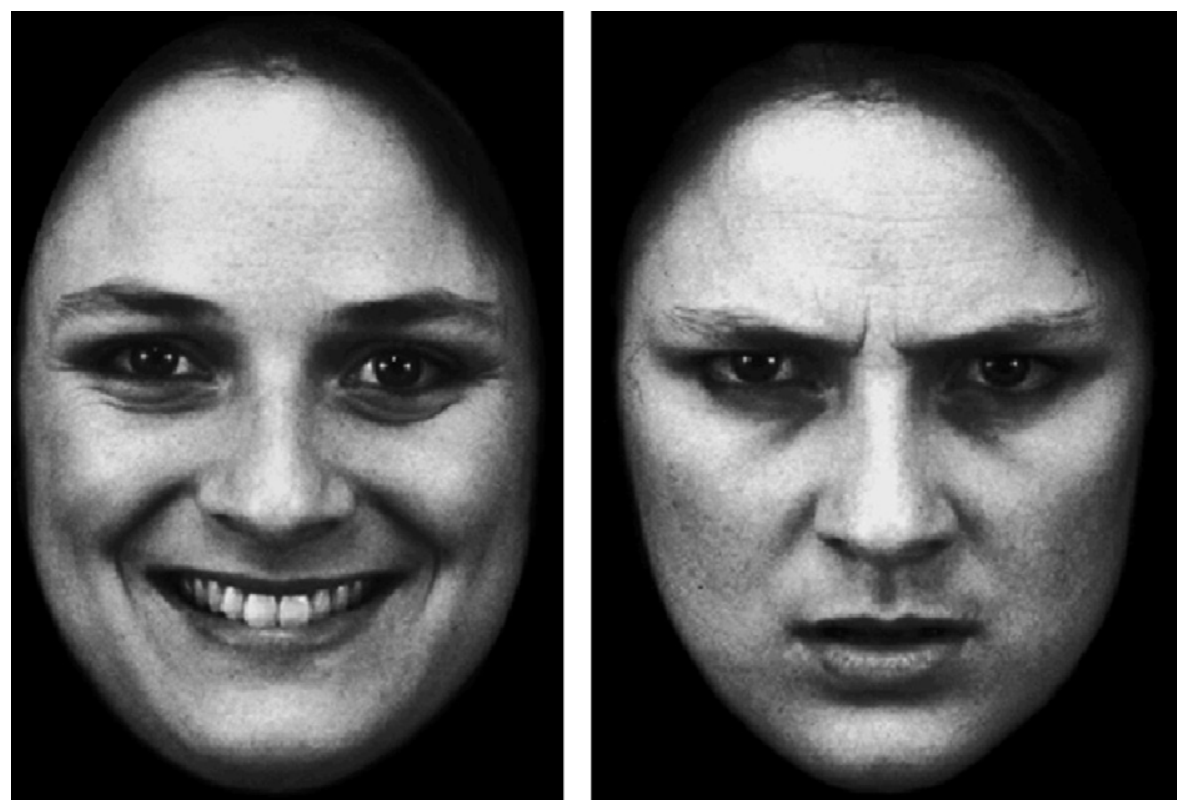

Fig. 1. Examples of a happy and angry face stimulus used in the AA task.

short recording ( $\sim 15 \mathrm{~min}$ ) of the electroencephalogram (EEG) during rest, after which the approach-avoidance task was administered, followed by a number of additional cognitive tests of which the results will be reported elsewhere. During task performance, participants sat in an air-conditioned and sound-attenuated room in front of a computer monitor, and the experimenter sat in an adjacent room, where the EEG apparatus was located.

\subsection{Approach-avoidance task}

In this affect-evaluation task (Rotteveel and Phaf, 2004), 60 pictures with facial expressions from Ekman and Friesen (1976), Matsumoto and Ekman (1988), and Lundqvist et al. (1998) served as stimuli. Half of the pictures were taken from female and the other half from male models (total of 30 models). Pictures consisted of grayscale photographs presented against a black background (see Fig. 1). To minimize variation in physical parameters unrelated to emotional expression, both the happy and the angry expression were taken from the same model. In addition, each face was trimmed to exclude the hair and nonfacial contours, and adjusted to match for size, brightness and contrast. Each picture measured $12.4 \mathrm{~cm} \times 8.9 \mathrm{~cm}(h \times w)$, and was presented at the center of a $15 \mathrm{in}$. computer screen at $70 \mathrm{~cm}$ viewing distance, resulting in a $10.1^{\circ} \times 7.3^{\circ}$ visual angle.

The start of an individual trial was indicated by the appearance of a central fixation point (lasting $100 \mathrm{~ms}$ ). After an interval of $300 \mathrm{~ms}$ the stimulus was presented for $100 \mathrm{~ms}$. The time interval between successive stimuli was randomized between 1500 and $2500 \mathrm{~ms}$. Pictures were presented using the Wesp Experimentation Stimulus Program (version 1.98 WESP XP, Molenkamp, University of Amsterdam, 2002).

Responses were registered by means of three buttons (of $16 \mathrm{~cm}^{2}$ ) that were fixed to a vertical stand (see Rotteveel and Phaf, 2004, Fig. 1). Participants sat to the left of the stand, allowing them to respond with their right hand. For the resting position participants were instructed to push the home button (fixed in the middle) loosely with the back of their right hand as long as no response was given. The height of this button was set for each participant individually, such that the angle between their forearm and upper arm was $110^{\circ}$ in the resting position. In this position both the biceps and the triceps were equally tensed. The response buttons were positioned above and below the home button (at a distance of $10.3 \mathrm{~cm}$ ). This allowed participants to simply flex or extend their right arm in responding without the need for precise aiming at the response buttons.

Participants were verbally instructed to evaluate the facial expressions (i.e. happy or angry), and to respond as fast and accurate as possible to the stimuli by releasing the home button and pressing one of the response buttons. After this, they had to return their hand to the home button. Participants received alternately an affect-congruent or an affect-incongruent instruction. The affect-congruent instruction indicated pressing the upper response button (i.e. arm flexion) for happy faces and the lower button (i.e. arm extension) for angry faces. In the affect-incongruent condition the mapping of the facial expression to the response buttons was reversed. No reference was made in the instructions to congruence and incongruence, approach and avoidance, or arm flexion and extension.

The task consisted of four series of 60 trials. Within each series all stimuli were presented once in a semi-randomized order (with a maximum of three happy or angry and three male or female pictures in succession). Half of the participants started with a series with an affect-congruent instruction, followed by a series with an affect-incongruent instruction, another affect-congruent instruction series, and a final affect-incongruent instruction series. The other half of the participants received the reversed order of instructions. Between each series participants performed an unrelated working memory task (digit span or spatial memory) that served to ease the transition from affect-congruent to affect-incongruent instruction or vice versa. Each of the four series was divided into three blocks of 20 trials, with a short break ( $\sim 30 \mathrm{~s})$ between blocks, and was preceded by 20 practice trials of stimuli that were not included in the experimental series.

The task provided three behavioral measures: error rates (percentage incorrect responses) and two reaction time (RT) measures. The initiation time (IT) is the time between stimulus onset and the release of the home button. The movement time (MT) is the time between the release of the home button and the pushing of the response button. IT constitutes an index of central processes reflecting stimulus evaluation, response selection and programming the execution of movements, and is relatively independent of MT, which reflects the magnitude of the neuro-muscular response (Fitts, 1954). The influence of affect on the reaction times is therefore primarily expected in IT, rather than MT (see Rotteveel and Phaf, 2004; Solarz, 1960). Incorrect responses and RTs that deviated more than 2.5 S.D. from the individual RT averages per cell (cells defined by cortisol condition $\times$ emotion $\times$ arm movement) were excluded from the RT analyses.

\subsection{Electrophysiological recording and analysis}

The electroencephalogram (EEG) was recorded from 19 scalp locations according to the international 10-20 system and referred on-line to $\mathrm{C} 3 / \mathrm{C} 4$. An average earlobe reference was derived off-line. Vertical electro-oculogram (EOG) was recorded bipolarly from the supraorbital and the infraorbital ridge of the right eye, and horizontal EOG from the outer canthi of both eyes. The 
ground electrode was located at Fpz. EEG impedances were kept below $5 \mathrm{k} \Omega$. The EEG and EOG signals were digitized at $500 \mathrm{~Hz}$. Signals were processed off-line using Brain Vision Analyzer software (version 1.05, Brain Products $\mathrm{GmbH}, 1998-2004)$. Codes synchronized to stimulus presentation and response were used to allow off-line averaging of epochs associated with specific stimulus and response types. The epoch ran for $1000 \mathrm{~ms}$, beginning $200 \mathrm{~ms}$ prior to stimulus onset, aligned to a $100 \mathrm{~ms}$ prestimulus baseline. Single trials were corrected for the effects of eye blinks and eye movements using a standard procedure (Gratton et al., 1983). Data were subsequently filtered digitally with a $0.1 \mathrm{~Hz}$ high-pass filter, a $35 \mathrm{~Hz}$ low-pass filter (both with a roll-off of $12 \mathrm{~dB} / \mathrm{oct}$ ) and a $50 \mathrm{~Hz}$ notch filter. After baseline correction, trials including amplitude values larger than $\pm 75 \mu \mathrm{V}$, a difference $>100 \mu \mathrm{V}$ between the lowest and the highest amplitude within the segment, a period $>100 \mathrm{~ms}$ with activity $<0.50 \mu \mathrm{V}$, or a difference $>50 \mu \mathrm{V}$ between two subsequent sampling points were considered artifacts and were excluded from analyses ( $9 \%$ of total dataset). We analyzed stimulus-locked data only for trials with correct responses with reaction times between 150 and $1000 \mathrm{~ms}$, computing averages for each category (defined by emotion $\times$ arm movement). After rejection of artifacts and incorrect responses a mean number of 49.7 trials (S.D. $=9.4$ ) per category was left for each participant in each cortisol condition for further analysis. To facilitate peak detection, individual averages per category were low-pass filtered at $12 \mathrm{~Hz}$ before peaks were identified and measured. The following stimulus-locked ERP components (peak amplitudes relative to baseline) were identified at electrodes F3, Fz, F4, C3, Cz, C4, P3, Pz, and P4: N1 (the first major negative wave occurring $30-130 \mathrm{~ms}$ post-stimulus), followed by $\mathrm{P} 150$ (the first major positive wave occurring $120-200 \mathrm{~ms}$ post-stimulus), N2 (180-300 ms), and P3 (270$400 \mathrm{~ms}$ ). Time windows for peak detection were based on visual inspection of the grand average ERPs, averaged across all participants and categories.

\subsection{Trait measures}

As described above, participants were assigned to two groups based on their score on the Behavioral Inhibition Scale (BIS). ${ }^{2}$ This seven-item self-report scale measures sensitivity to signals of threat and was shown to have good reliability (BIS/BAS: Carver and White, 1994). Items are statements that reflect a concern over the possibility of a bad occurrence or a sensitivity to such events when they do occur, and each item is rated on a four-point scale, with a maximum total score of 28. The Behavioral Activation Scale (BAS) consists of a total of 13 items measuring sensitivity to reward. In addition, we administered questionnaires measuring trait anxiety (State Trait Anxiety Inventory (STAI): Spielberger, 1983; Dutch version: Van der Ploeg, 2000) and social anxiety (Social Phobia and Anxiety Inventory (SPAI): Turner et al., 1989; Dutch version: Bögels and Reith, 1999), as well as the temperament subscales of the Temperament and Character Inventory (TCI), which contains a Novelty Seeking and Harm Avoidance subscale that have been related to behavioral activation and behavioral inhibition, respectively (Cloninger et al., 1994; Dutch version: De la Rie et al., 1998).

\subsection{Cortisol and subjective measures}

Saliva samples were obtained using Salivette collection devices (Sarstedt, Rommelsdorf, Germany). Samples were obtained at four assessment points over a $165 \mathrm{~min}$ period, at respectively $-5 \mathrm{~min}(\mathrm{~T} 0),+60 \mathrm{~min}(\mathrm{~T} 1),+120 \mathrm{~min}(\mathrm{~T} 2)$, and +160 min (T3) with reference to capsule ingestion. Biochemical analysis of free cortisol in saliva was performed using a competitive electrochemiluminescence immunoassay (ECLIA, Elecsys 2010, Roche Diagnostics), as described elsewhere (Van Aken et al., 2003).

Self-reported mood (tension, fatigue, depression, anxiety, and activation at $\mathrm{T} 0, \mathrm{~T} 1$, and $\mathrm{T} 3$ ) and motivation and concentration (directly before and after the

\footnotetext{
${ }^{2}$ The BIS/BAS scales of Carver and White (1994) were developed on the basis of the reinforcement sensitivity theory (RST: e.g. Gray, 1982). Note that due to a recent revision of this theory (Gray and McNaughton, 2000) the BIS scale, designed to measure threat sensitivity, is likely associated with the Fight Flight and Freezing System in the revised RST (Smillie et al., 2006).
}

AA-task) were rated on $10 \mathrm{~cm}$ visual analogue scales (VAS). In addition, state anxiety (STAI-state: Spielberger, 1983) was measured at T0 and T3.

\subsection{Statistical analyses}

The influence of cortisol administration on subjective measures, salivary cortisol, AA-task performance, and ERP peak amplitudes were tested with repeated measures analyses of variance (ANOVAs $\mathrm{rm}$ ) using the Statistical Package for the Social Sciences (SPSS 14.0, SPSS Inc., 1989-2005). All statistical analyses described employed a two-tailed alpha of .05. Effect sizes are reported as proportion of explained variance (partial eta squared $\left[\eta^{2}\right]$ ). Reaction times of two participants (both from the low BIS group) were not registered due to technical problems. These participants were excluded from all analyses, resulting in a total number of 18 subjects in the low BIS group.

\section{Results}

\subsection{Trait measures}

Table 1 presents the mean values for the low and the high BIS groups on the trait measures. As expected, and due to our selection procedure, groups differed significantly on BIS-scores $\left(F(1,36)=177.87, p<.001, \eta^{2}=0.83\right)$. In addition, the high BIS group scored significantly higher on several anxiety measures: trait anxiety (STAI-T: $F(1,36)=30.18, p<.001$, $\left.\eta^{2}=0.46\right)$, social anxiety (SPAI total: $F(1,35)=11.31$, $p<.01, \quad \eta^{2}=0.26$ ) and harm avoidance (TCI-HA: $F(1$, $\left.36)=26.01, p<.001, \eta^{2}=0.42\right)$. The groups did not differ significantly in age, body mass index or any of the other trait measures (all $p>.10)$.

\subsection{Cortisol and subjective measures}

\subsubsection{Salivary cortisol}

Salivary cortisol (nmol/L) measures (see Table 2) were skewed and therefore $\log$ transformed before statistical analysis. The results of a 2 (group: low BIS, high BIS) $\times 2$ (cortisol: placebo, cortisol) $\times 4$ (time: T0, T1, T2, T3) ANOVA rm yielded a significant interaction of cortisol $\times$ time $(F(3,102)=188.92$,

Table 1

Trait scores for low BIS and high BIS groups

\begin{tabular}{|c|c|c|c|c|}
\hline \multirow[t]{2}{*}{ Measure } & \multicolumn{2}{|c|}{ Low BIS } & \multicolumn{2}{|c|}{ High BIS } \\
\hline & $M$ & S.D. & $M$ & S.D. \\
\hline Age & 20.4 & 1.7 & 19.9 & 1.4 \\
\hline BMI & 21.4 & 1.6 & 21.6 & 1.7 \\
\hline BIS $^{* * *}$ & 14.0 & 2.1 & 22.1 & 1.7 \\
\hline BAS total & 37.9 & 4.4 & 37.9 & 3.4 \\
\hline STAI-trait ${ }^{* * *}$ & 29.6 & 4.9 & 37.6 & 4.1 \\
\hline SPAI total ${ }^{* *}$ & 40.4 & 17.5 & 57.8 & 13.0 \\
\hline \multicolumn{5}{|l|}{ TCI } \\
\hline Harm avoidance ${ }^{* * * *}$ & 4.1 & 3.4 & 9.4 & 3.0 \\
\hline Novelty seeking & 9.5 & 4.2 & 8.9 & 3.4 \\
\hline Reward dependence & 8.7 & 2.5 & 10.0 & 2.7 \\
\hline Persistence & 1.8 & 1.6 & 2.2 & 1.2 \\
\hline
\end{tabular}

Note : BMI = body mass index; BIS = Behavioral Inhibition Scale; BAS = Behavioral Activation Scale; STAI = State Trait Anxiety Inventory; SPAI = Social Phobia and Anxiety Inventory; TCI $=$ Temperament and Character Inventory. *** $p<.01$. **** $p<.001$ 
Table 2

Mean free salivary cortisol levels (nmol/L) after placebo and cortisol administration relative to time of capsule intake $(t=0)$

\begin{tabular}{clllrr}
\hline Time (min) & \multicolumn{2}{l}{ Placebo } & & \multicolumn{2}{l}{ Cortisol } \\
\cline { 2 - 3 } \cline { 5 - 6 } & $M$ & S.D. & & $M$ & S.D. \\
\hline-5 & 8.9 & 3.2 & & 9.1 & 2.8 \\
$+60^{* * *}$ & 6.8 & 1.7 & & 161.7 & 145.1 \\
$+120^{* * * *}$ & 6.7 & 3.5 & & 122.5 & 55.0 \\
$+165^{* * *}$ & 6.5 & 2.6 & & 112.4 & 50.2 \\
\hline
\end{tabular}

Note: $N=36$ due to missing values of two participants.

**** $p<.001$.

$\left.p<.0001, \eta^{2}=0.98\right)$. This result indicates that, as expected, unbound levels of cortisol did not differ between conditions before capsule intake (T0: $F(1,35)=0.44, p=.51$ ), but were significantly increased after cortisol administration compared to placebo from $1 \mathrm{~h}$ after capsule intake $(\mathrm{T} 1: F(1,35)=320.48$, $\left.p<.0001, \eta^{2}=0.90\right)$ until the end of the experiment (T2: $F(1$, $34)=846.67, p<.0001, \eta^{2}=0.96$; T3: $F(1,35)=1265.77$, $\left.p<.0001, \eta^{2}=0.97\right)$. There were no significant differences in salivary cortisol values between groups.

\subsubsection{Subjective measures}

To investigate group differences in subjective mood during task administration and effects of cortisol administration on mood, we conducted separate ANOVAs rm with group (low BIS, high BIS) $\times$ cortisol (placebo, cortisol) $\times$ time for STAIstate (T0, T3) and VAS (T0, T1, T3) tension, fatigue, depression, anxiety, and activation. Results showed significant main effects of group on STAI-state anxiety $(F(1,36)=8.49$, $\left.p<.01, \eta^{2}=0.19\right)$ and VAS tension $(F(1,36)=7.23, p<.05$, $\left.\eta^{2}=0.17\right)$ indicating higher scores for the high BIS group (STAI-S: $M=33.5$; TEN: $M=2.7$ ) compared to the low BIS group (STAI-S: $M=28.8$; TEN: $M=1.9$ ). In addition, VAS anxiety scores tended to be higher for high BIS $(M=1.6)$ compared to low BIS $(M=1.3)$ participants $(F(1,36)=4.06$, $\left.p=.051, \eta^{2}=0.10\right)$. There were no significant main or interaction effects of cortisol on mood.

\subsection{Behavioral results}

To investigate the influence of cortisol administration and trait avoidance on performance of the AA-task we conducted separate 2 (group: low BIS, high BIS) $\times 2$ (cortisol: placebo, cortisol) $\times 2$ (emotion: happy, angry) $\times 2$ (arm movement: approach (flex), avoid (extend)) ANOVAs rm on error rates and reaction times (MT and IT). ${ }^{3}$ For all behavioral measures we will first present results concerning the AA congruency-effect (i.e. the emotion $\times$ arm movement interaction) and subsequently the effects of group and cortisol on this congruency effect.

\footnotetext{
${ }^{3}$ We performed two additional analyses, with session (first day, second day) and stimulus gender (male, female) as additional factors. Since both analyses revealed no significant effects of these factors on the emotion $\times$ arm movement interaction, we have further left them out of the analyses.
}

\subsubsection{Error rates}

As to be expected in the AA-task (see Rotteveel and Phaf, 2004), a significant emotion $\times$ arm movement interaction $\left(F(1,36)=5.77, p<.05, \eta^{2}=0.14\right)$ showed that participants made more errors $(\%)$ during affect-incongruent arm movements (avoid happy: $M=7.2$; approach angry: $M=8.8$ ) than affect-congruent arm movements (approach happy: $M=5.3$; avoid angry: $M=7.8$ ). There were no effects of group or cortisol on these congruency effects (all $p>.16)$.

\subsubsection{Initiation times (IT)}

Also for the IT (in ms), we found the expected AA congruency effect. A significant emotion $\times$ arm movement interaction $\left(F(1,36)=21.05, p<.0001, \eta^{2}=0.37\right)$ showed that participants were faster in initiating affect-congruent (approach happy: $M=486$; avoid angry: $M=500$ ) than affect-incongruent arm movements (avoid happy: $M=506$; approach angry: $M=511)$. The effects of group or cortisol on this emotion $\times$ arm movement interaction were not significant.

We did, however, find a significant three-way interaction of group $\times$ cortisol $\times$ arm movement $(F(1,36)=15.03$, $\left.p<.0001, \eta^{2}=0.29\right)$. Separate ANOVAs for the high and low BIS group showed a significant cortisol $\times$ arm movement interaction for the high BIS group $(F(1,19)=16.11$, $\left.p<.001, \eta^{2}=0.46\right)$, but not for the low BIS group $(F(1$, $17)=2.25, p=.15$ ) (see Fig. 2). The significant results for the high BIS group were due to a significant effect of cortisol on the approach movement $(F(1,19)=5.76, p<.05$, $\left.\eta^{2}=0.23\right)$, indicating that approach reactions were slowed after cortisol administration in high BIS participants. The cortisol $\times$ emotion $\times$ arm movement interaction was not significant in the high BIS group $(F(1,19)=0.38$, $p=.55)$, indicating that the cortisol $\times$ arm movement interaction was not different for happy and angry faces. However, because we had specific hypotheses about this effect for angry faces, we additionally checked whether the cortisol $\times$ arm movement interaction for the high BIS group would hold when tested for responses to angry faces only. The results indeed showed the cortisol $\times$ arm movement interaction in the high BIS group to be significant for angry faces $\left(F(1,19)=10.30, p<.01, \eta^{2}=0.35\right)$. Interestingly, this effect was not significant for happy faces (cortisol $\times$ arm movement: $F(1,19)=2.16, p=.16)$. In addition, due to the slowing of approach reactions after cortisol administration, the congruency effect for angry faces (i.e. faster avoidance than approach) was only significant for the high BIS group in the cortisol condition (arm movement: $F(1,19)=8.84$, $\left.p<.01, \eta^{2}=0.32\right)$ and not the placebo condition $(F(1$, 19) $=0.17, p=.69$ ) (see Fig. 2).

Thus, in line with our hypotheses, cortisol administration affected approach-avoidance congruency effects especially to angry faces in high BIS participants. Although the results indicated that this effect did not differ significantly between happy and angry faces, the effect was only significant for angry, and not for happy faces. 

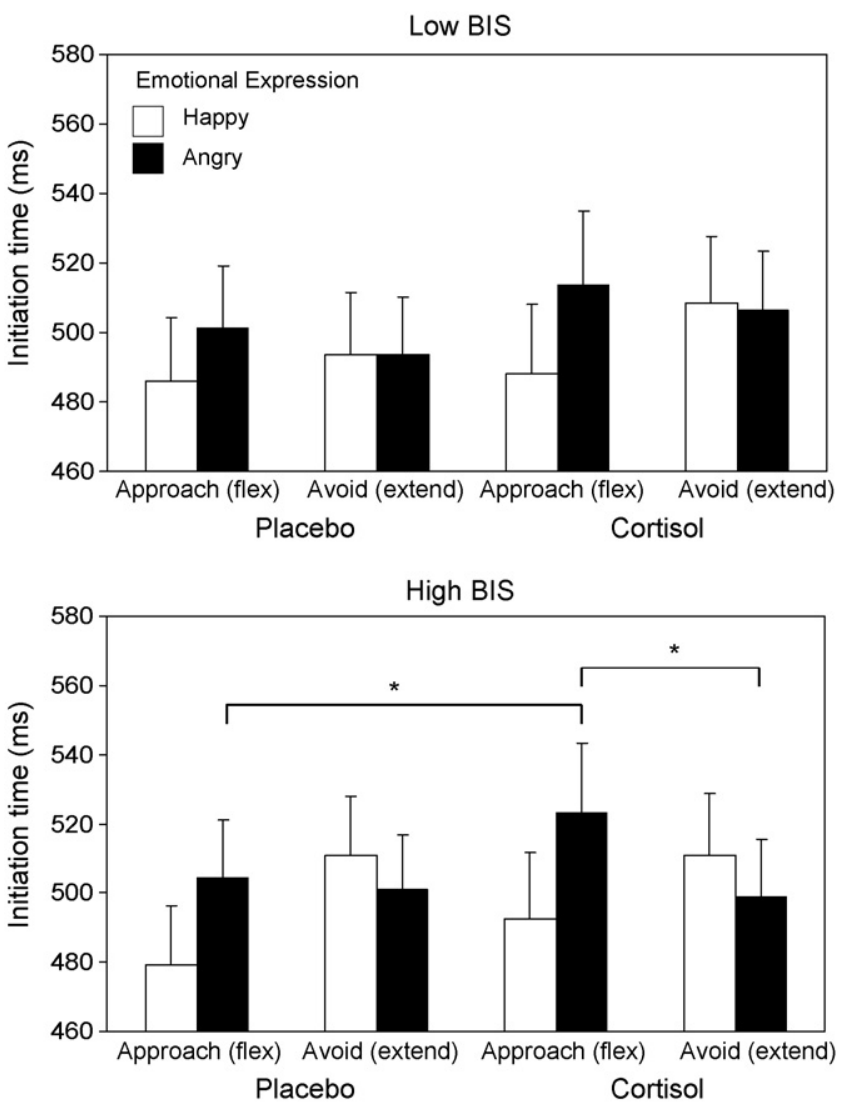

Fig. 2. Mean initiation times (in ms) on the AA task for the low BIS (upper panel) and high BIS (lower panel) group after placebo and cortisol administration. Cortisol administration resulted in a significant slowing of approach, but not avoidance, movements in the high BIS group only. This effect was significant for angry faces, but not for happy faces. The congruency effect for angry faces (i.e. faster avoidance than approach) was only significant in the high BIS group after cortisol administration. Error bars indicate the standard errors of the means. ${ }^{*} p<.05$.

\subsubsection{Movement times (MT)}

Like the error rates and IT, the MT (in ms) showed a significant emotion $\times$ arm movement interaction $(F(1$, $\left.36)=8.48, p<.01, \eta^{2}=0.19\right)$ indicating faster execution of affect-congruent (approach happy: $M=138$; avoid angry: $M=134$ ) than affect-incongruent arm movements (avoid happy: $M=140$; approach angry: $M=142$ ). There were no effects of group or cortisol on these congruency effects in MT.

\subsection{Event-related potentials}

The data from the $\mathrm{Cz}$ electrode appeared most representative for the three midline electrodes $(\mathrm{Fz}, \mathrm{Cz}, \mathrm{Pz})$ and are presented in Fig. 3. The general morphology of the waveform at these midline electrodes included a prominent, early negative peak at $100 \mathrm{~ms}$ (N1), followed by a positive wave at $150 \mathrm{~ms}$ (P150), a second negative wave at $230 \mathrm{~ms}$ (N2) and a final positive wave at $350 \mathrm{~ms}$ (P3). As shown in Fig. 3, event-related peaks were pronounced.

Baseline-to-peak amplitudes were analyzed with separate 2 (group: low BIS, high BIS) $\times 2$ (cortisol: placebo, cortisol) $\times 2$ (emotion: happy, angry) $\times 2$ (arm movement: approach (flex), avoid (extend) $\times 3$ (electrode: $\mathrm{Fz}, \mathrm{Cz}, \mathrm{Pz})^{4}$ ANOVAs $\mathrm{rm}$ for $\mathrm{N} 1, \mathrm{P} 150, \mathrm{~N} 2$, and $\mathrm{P} 3$. As for the behavioral results, we will focus on the AA congruency-effects (i.e. the emotion $\times$ arm movement interaction) and the effects of group and cortisol on this interaction.

\subsection{1. $P 150$}

For P150 peak amplitude, we found a significant four-way interaction of group $\times$ cortisol $\times$ emotion $\times$ arm movement $\left(F(1,36)=4.94, p<.05, \eta^{2}=0.12\right)$. Follow up analyses to determine the nature of this interaction showed that the emotion $\times$ arm movement interaction was only significant for the high BIS group in the cortisol condition $(F(1,19)=6.50$, $p<.05, \eta^{2}=0.26$ ) (see Fig. 4, panel A). It was not significant for the high BIS group in the placebo condition $(F(1$, $19)=0.17, p=.69)$, nor was it significant for the low BIS group in either the cortisol condition $(F(1,17)=1.88$, $p=.19)$ or the placebo condition $(F(1,17)=0.42, p=.53)$. Further analyses of this emotion $\times$ arm movement interaction for the high BIS group in the cortisol condition revealed that the effect of arm movement was significant for angry faces $\left(F(1,19)=9.93, p<.01, \eta^{2}=0.34\right)$, but not for happy faces $(F(1,19)=0.68, p=.42)$. This indicates that only in response to angry faces P150 amplitude was significantly higher (i.e. more positive) when these participants made an avoidant arm movement, than when they made an approaching arm movement. Thus, consistent with the behavioral (IT) results, we found significant congruency effects (i.e. approach versus avoidance) for the high BIS group in the cortisol condition for angry faces only. Also, the P150 amplitude of high BIS participants in the cortisol condition was significantly higher in response to angry compared to happy faces only for avoidant arm movements (emotion: $F(1,19)=14.15$, $p<.001)$, and not for approaching arm movements $(F(1$, 19) $=0.00, p=.99)$.

\subsubsection{P3}

For P3 amplitude, the group $\times$ cortisol $\times$ emotion $\times$ arm movement ANOVA rm yielded a significant three-way interaction of group $\times$ cortisol $\times$ arm movement $(F(1,36)=$ $\left.5.13, p<.05, \eta^{2}=0.13\right)$ and a significant three-way interaction of cortisol $\times$ emotion $\times$ arm movement $(F(1,36)=4.13$, $\left.p<.05, \eta^{2}=0.10\right)$. Although post hoc analyses of the first interaction did not reveal significant effects, post hoc analyses for the cortisol $\times$ emotion $\times$ arm movement interaction showed the following effects: The emotion $\times$ arm movement interaction was significant in the cortisol condition $(F(1$, $\left.36)=5.78, p<.05, \eta^{2}=0.14\right)$, but not in the placebo condition $(F(1,36)=0.00, p=.98)$. In the cortisol condition, the P3 amplitude for avoidant arm movements was significantly higher

\footnotetext{
${ }^{4}$ A second analysis for each component was conducted with F3, F4, C3, C4, $\mathrm{P} 3$, and $\mathrm{P} 4$ as additional electrodes, and with laterality (left, midline, right) as an additional factor. This analysis confirmed the conclusion based on visual inspection that there were no laterality effects involving emotion $\times$ arm movement. Therefore, only results of midline electrodes are presented.
} 

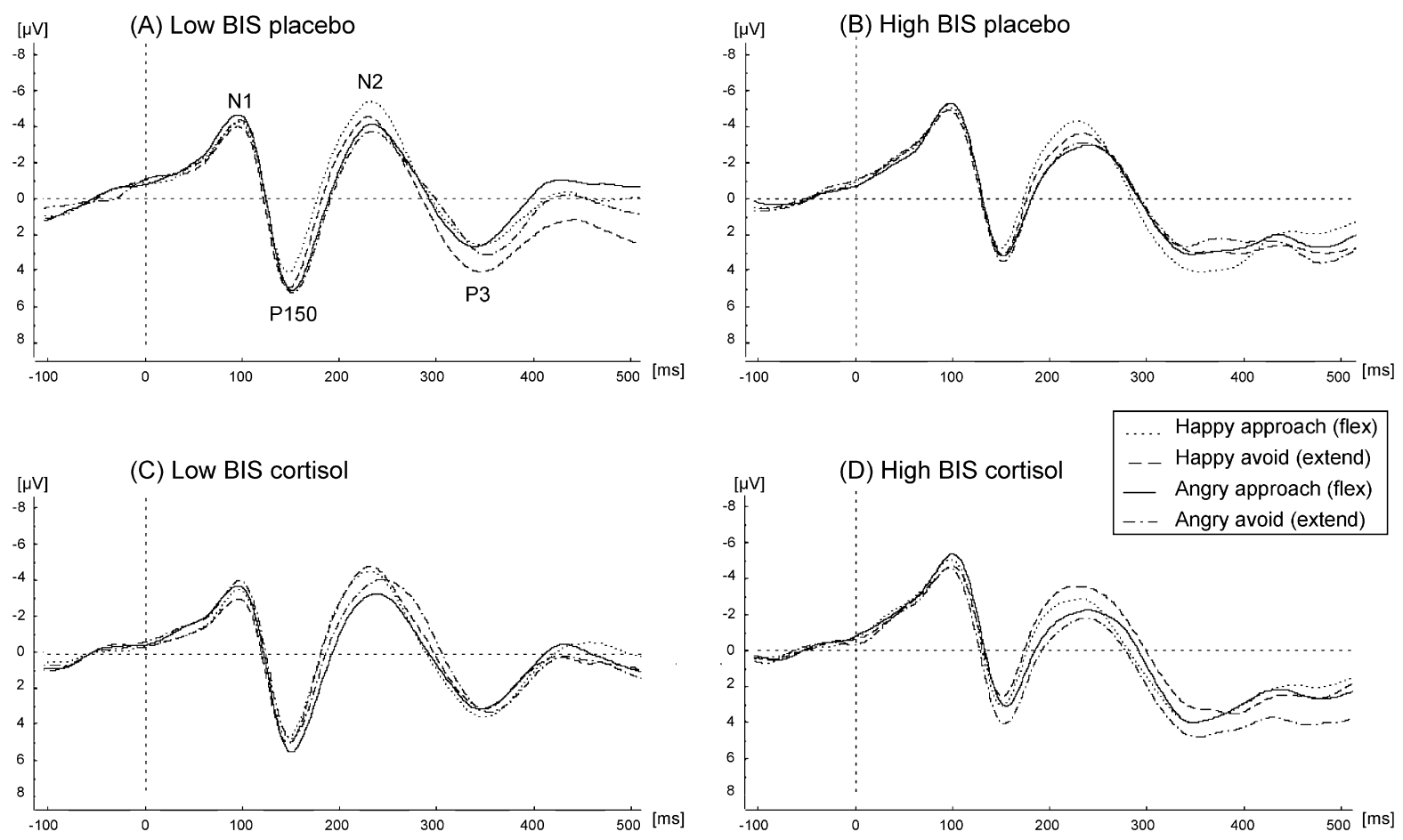

Fig. 3. Stimulus synchronized event-related potential (ERP) waveforms at $\mathrm{Cz}$ for the low BIS (panels A and C) and high BIS (panels B and D) groups after placebo (panels A and B) and cortisol (panels C and D) administration. Stimulus onset was at $t=0$. Lines represent the categories defined by the emotional expression of the stimuli and the arm movement of the response.

(i.e. more positive) in response to angry faces than in response to happy faces (emotion: $F(1,36)=8.85, p<.01, \eta^{2}=0.20$ ). The four-way group $\times$ cortisol $\times$ emotion $\times$ arm movement interaction was not significant $(F(1,36)=0.02, p=.88)$, indicating that this effect did not differ between groups. However, because we had specific hypotheses about the congruency effects after cortisol administration in the high BIS participants, we additionally checked whether the emotion $\times$ arm movement interaction in the cortisol condition would hold when tested in the high BIS group only. The results indeed indicated that the emotion $\times$ arm movement interaction in the cortisol condition was significant for the high BIS group only $\left(F(1,19)=4.67, p<.05, \eta^{2}=0.20\right)$. Interestingly, it was not significant for the low BIS group $(F(1,17)=1.45, p=.25)$, nor was it significant for either group in the placebo condition (low BIS: $F(1,17)=0.57, p=.46$; high BIS: $F(1,19)=0.72$, $p=.41$ ). Follow up analyses indicated that, in line with the P150 results, for the high BIS group in the cortisol condition the effect of Emotion was significant for avoidant arm movements $\left(F(1,19)=12.67, p<.003, \eta^{2}=0.40\right)$, but not for approaching arm movements $(F(1,19)=0.86, p=.37)$. Thus, $\mathrm{P} 3$ amplitudes were significantly higher in response to angry faces than in response to happy faces only when an avoidant arm movement was made by high BIS individuals in the cortisol condition (see Fig. 4, panel B).

\subsubsection{N1 and N2}

We did not find a significant emotion $\times$ arm movement interaction for $\mathrm{N} 2$ amplitude $(F(1,36)=0.15, p=.70)$, indicating that $\mathrm{N} 2$ amplitude was not increased for affect- incongruent arm movements (avoidance of happy faces and approach of angry faces) compared to affect-congruent arm movements (approach of happy faces and avoidance of angry faces).

Although we did not have specific expectations about possible effects of cortisol and group on congruency effects for the N2, visual inspection of Fig. 3 suggested that N2 amplitudes showed similar effects as P150 and P3. This may suggest a general effect of cortisol administration on ERP amplitudes in the high BIS group. Indeed, significant N2 congruency effects were found in the high BIS group after cortisol administration only (emotion $\times$ arm movement: $F(1,19)=11.55, p<.01$, $\eta^{2}=0.38$ ) (see Fig. 4, panel C). Thus, although the effects of cortisol administration on the N2 congruency effects were not significant in the four-way ANOVA (group $\times$ cortisol $\times$ emotion $\times$ arm movement: $F(1,36)=2.38, p=.13)$, the $\mathrm{N} 2$ effects showed trends in the same direction as the IT and positive ERP wave results. As expected we found no other significant effects involving emotion and arm movement on negative waves (N1: all $p>.20)$.

\section{Discussion}

With the present study we aimed to investigate the influence of cortisol administration on approach and avoidance behavior towards positive and negative social stimuli in high and low avoidant participants (i.e. scoring high or low on the Behavioral Inhibition Scale (BIS)). The second aim was to investigate the associated brain processes using ERPs, with specific focus on components involved in emotional face processing and action 

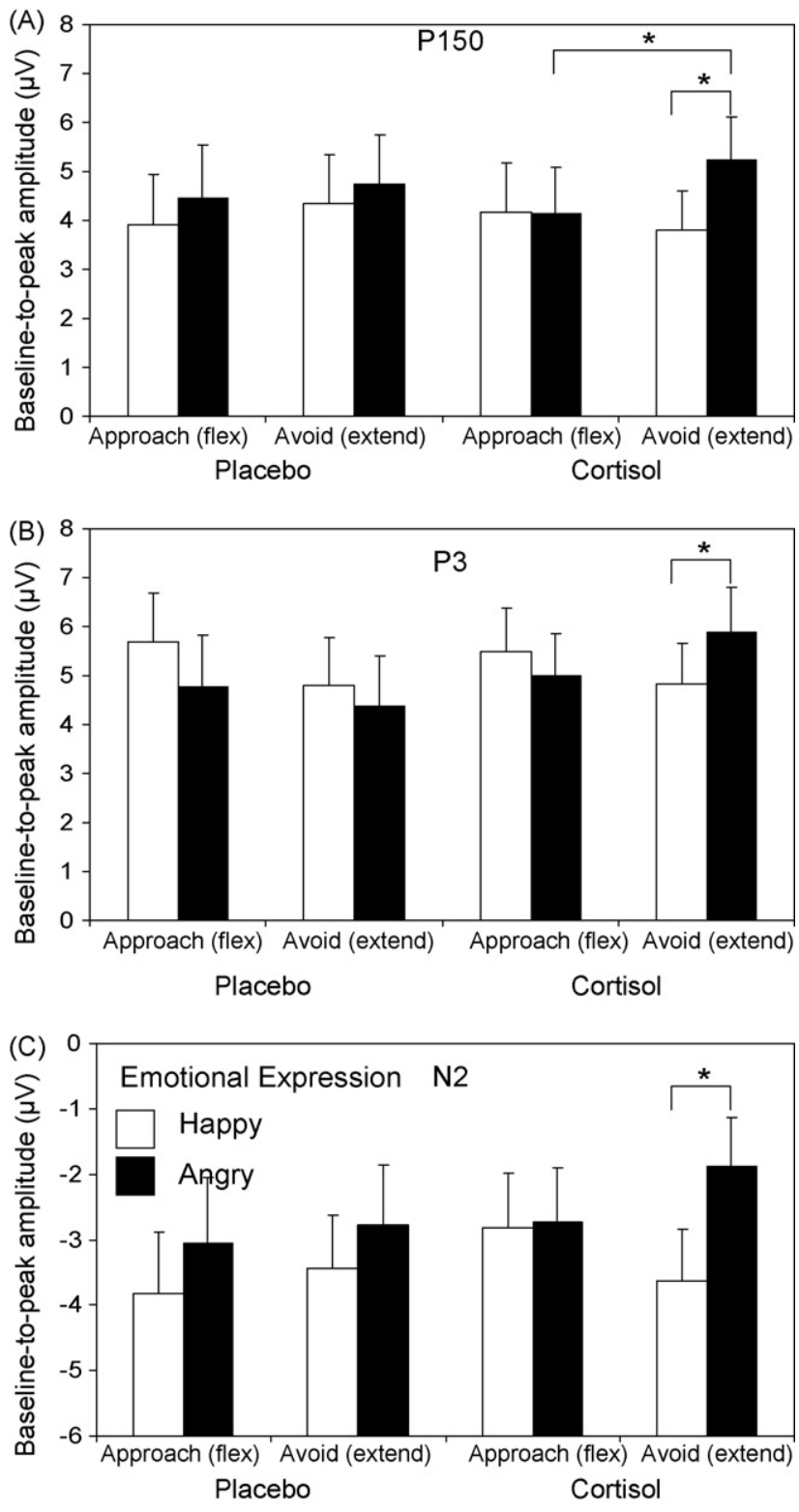

Fig. 4. Baseline-to-peak amplitude (in $\mu \mathrm{V}$ ) for P150 (panel A), P3 (panel B), and N2 (panel C) ERP components for the high BIS group after placebo (left) and cortisol (right) administration. All three components show a significant emotion $\times$ arm movement interaction in the high BIS group after cortisol administration only, with most positive amplitudes in response to angry faces when an affect-congruent avoidance response (arm extension) is made. Note that for the $\mathrm{N} 2$ component (panel C) the values of the $y$-axis are inverted, such that consistent with panels A and B a higher bar indicates a more positive amplitude. Error bars indicate the standard errors of the means. ${ }^{*} p<.05$.

monitoring. Compared to low BIS participants, we expected high BIS participants to show relatively increased threat avoidance, and we expected that cortisol administration would result in a facilitation of this threat avoidance. In addition, these behavioral effects were hypothesized to be accompanied by increased amplitudes of ERP components involved in motivational processes. Our results were largely in line with our expectations, showing cortisol administration in high BIS participants to result in enhanced AA congruency effects in both initiation times and positive ERP amplitudes for angry faces. Below, these behavioral and ERP results will be first discussed separately. Thereafter, these results will be integrated in the light of previous findings related to glucocorticoid effects on cognition and threat processing.

\subsection{Behavioral results}

First, consistent with previous findings (Chen and Bargh, 1999; Roelofs et al., 2005; Rotteveel and Phaf, 2004), this study showed the expected congruency effects, as reflected by faster initiation times (IT), faster movement times (MT) and less errors for affect-congruent (i.e. approach happy and avoid angry faces) compared to affect-incongruent (avoid happy and approach angry faces) arm movements.

In addition, in the high BIS, but not the low BIS group, cortisol administration resulted in a significant slowing of approach reactions (IT). In line with our expectations, this resulted in a significant increase of the approach-avoidance congruency effect for angry faces (faster avoidance than approach), but not for happy faces. However, these differential effects for valence should be interpreted with caution. The lack of a four-way interaction including the emotional valence of the faces suggests an inhibition of approach reactions to social stimuli, independent of stimulus valence. However, our present interpretation is supported by the ERP results (as will be discussed later), which do suggest a differential effect of cortisol administration on processing of happy and angry faces.

In spite of the fact that our groups were a priori selected on the basis of extreme high or low BIS scores, and differed significantly with respect to trait anxiety and social anxiety, they did not differ in approach-avoidance reactions in the placebo condition. This may be due to the fact that all participants were healthy students. It is also important to note that basal cortisol levels did not differ between high and low BIS participants. Apparently the approach-avoidance reactions of high and low BIS participants differed only after cortisol administration. These results are consistent with the findings of Roelofs et al. (2005), who also found no differences between high and low trait avoidant individuals (based on a post hoc median-split on BIS scores) on approach-avoidance behavior, independent of cortisol.

Finally, consistent with previous findings (Roelofs et al., 2005), we did not find significant effects of BIS group or cortisol on the error rates or the movement times (MT). This is not surprising, since participants generally make few errors in this task, and MT has been suggested to be predominantly affected by physical parameters of movement, and not by central cognitive processes (see Rotteveel and Phaf, 2004).

\subsection{ERP results}

The second purpose of this study was to investigate the brain processes associated with approach and avoidance of happy and angry faces. In line with the behavioral results, we found a significant effect of cortisol administration on ERPs for high BIS participants only. After cortisol administration P150 amplitude was highest (i.e. most positive) in reaction to angry 
faces when high BIS participants made an avoidant arm movement. A similar effect was found on P3 amplitude, showing significantly higher positive amplitudes in reaction to angry as compared to happy faces when an avoidant arm movement was made by high BIS participants after cortisol administration. Although the lack of a four-way interaction including group (low BIS, high BIS) on P3 amplitude indicated that this effect did not differ significantly between groups, separate analyses for each group confirmed our specific a priori expectation that $\mathrm{P} 3$ amplitudes in reaction to angry faces would be particularly pronounced for high BIS participants after cortisol administration, given the increased motivational significance of threat stimuli for these participants.

Increased amplitudes of early as well as late positive ERP components have been interpreted as reflecting increased allocation of processing resources to motivationally significant input (Eimer et al., 2003; Nieuwenhuis et al., 2005). The timing of the early effect in the present study (i.e. P150 amplitude) is in line with results of previous studies showing differential processing of faces signaling threat (i.e. fearful or threatening faces, 110-220 ms post-stimulus: e.g. Bar-Haim et al., 2005; Eimer and Holmes, 2002; Schupp et al., 2004; Williams et al., 2006), and suggests an effect on relatively early stages of information processing. Interestingly, data from single-neuron recordings in human ventromedial prefrontal cortex showed differential processing of threatening emotional face stimuli in the same time range (120-170 ms: Kawasaki et al., 2001), suggesting that early aspects of perceptual processing may be modulated via topdown influences, facilitating early identification of, and appropriate behavioral responses to, threat (see Bar, 2003).

The increased P3 amplitude in the present study is consistent with results of other studies indicating increased amplitudes of late positive components for emotionally negative or threat stimuli (e.g. Huang and Luo, 2006; Schupp et al., 2004), which are assumed to reflect more elaborate sustained perceptual processing of relevant emotional stimuli, via top-down influences from limbic and/or frontal areas (Eimer et al., 2003; Krolak-Salmon et al., 2001; Sato et al., 2001). Interestingly, we found enhanced P150 and P3 amplitudes for angry faces after cortisol administration only in high avoidant (high BIS) individuals, indicating that processing of angry faces after cortisol administration was specifically enhanced in individuals sensitive to threat. In addition, here the P150 amplitude for angry faces was significantly higher when an (affect-congruent) avoidance movement was made than when an (affect-incongruent) approach movement was made. This finding may be explained by the fact that the affectcongruent and affect-incongruent arm movements were blocked in separate instruction conditions, which may have strengthened the response mode within each condition, resulting in priming of affect-congruent stimulus processing.

On a more exploratory basis, we also tested whether the AA task could elicit significant N2 effects, reflected by increased amplitudes for affect-incongruent relative to affect-congruent arm movements. We did not find such effects. In other more frequently used paradigms involving congruent and incongruent stimulus-response mapping, such as Flanker or Stroop tasks, an N2 congruency effect is observed ubiquitously (see e.g. Yeung et al., 2004). A possible explanation for the discrepancy of our finding is related to the type of conflict that may be elicited by the AA task. In this task, the response conflict in incongruent trials is not the result of two competing endogenous responses elicited simultaneously by the stimulus, as is the case in Flanker and Stroop tasks, but results from a conflict between the instructed response and the intuitive response tendency elicited by the stimulus. Conflict or incompatibility in the AA task may therefore be represented at another level than in typical conflict tasks, and as a result it may not be reflected by increased N2 amplitudes. At present, the representational level at which action or conflict monitoring by the ACC takes place is still unclear (Van Veen et al., 2004).

\subsection{Cortisol effects and threat sensitivity}

Together, the behavioral and ERP findings showed cortisol administration to be associated with enhanced AA congruency effects in reaction to angry faces in high avoidant, but not low avoidant, individuals. However, whereas this effect was manifested in a slowing of affect-incongruent (approach) responses in behavior, ERPs showed enhanced positive amplitudes for affect-congruent (avoidance) responses.

The results of this study did not show a general effect of cortisol on approach and avoidance (AA) tendencies. Instead, the effects of cortisol administration on affect-congruent processing of, and initiation times to, angry faces in particular were mediated by individual differences in self-reported threat sensitivity (BIS). This finding may be viewed as consistent with the findings of Roelofs et al. (2005) who investigated the effects of stress-induced cortisol responses on AA behavior, using the same paradigm. In that study, the effects of stress-induction on approach and avoidance tendencies (IT) were found to be mediated by individual differences in cortisol responsiveness, which is possibly associated with individual differences in the tendency to perceive and respond to affective stimuli (Roelofs et al., 2005).

These results are also in agreement with the findings from animal studies showing that corticosteroid effects on cognition are context dependent, and are influenced by factors such as environmental input and concurrent information processing (De Kloet et al., 1999). People with high BIS scores are suggested to be especially responsive to threat cues (Carver and White, 1994) and thus may have a processing bias for threatrelated facial expressions, as has been previously found with anxious individuals (e.g. Bar-Haim et al., 2005; Fox et al., 2002; Mogg and Bradley, 2002). This processing bias has been found to increase under stressful conditions (Mathews and Macleod, 1994), as well as after acute cortisol administration in healthy young males (Putman et al., 2007). In the present study, the effects of cortisol administration may have interacted with a processing bias of threatening stimuli in high BIS participants. This interpretation is in line with the results of a study by Cools et al. (2005), who found that a manipulation of serotonin function interacted with individual differences in BIS scores to bias the processing of threatening stimuli. 
The present study is the first to show effects of cortisol administration on human approach-avoidance behavior, and several related questions remain unanswered. First, it remains to be explored whether the present findings are dose dependent. Second, the effects of cortisol administration in our study do not mimic the behavioral effects of high endogenous cortisol levels during stress, which were found to result in decreased approach and avoidance tendencies (Roelofs et al., 2005). This difference may be explained by the results of several recent studies (e.g. Roozendaal et al., 2004; Elzinga and Roelofs, 2005) suggesting that the impairing effects of cortisol on prefrontal functions depend on concurrent noradrenergic activation, which is present during stress, but not in our study. Interesting in this respect is that, consistent with the findings of the present study, Van Honk et al. (1998) found increased (basal) cortisol levels to be associated with increased avoidance of angry faces on an emotional Stroop task, when testing subjects in a non-stress condition. Taken together, these findings suggest an important role for the context in which cortisol levels are elevated. Future studies in which the effects of endogenous cortisol are attenuated, for example with the use of selective steroid receptor antagonists, may help to further assess the role of cortisol and the interplay with contextual effects in human cognition (De Kloet et al., 1999).

Third, since negative laboratory stimuli are routinely judged to be more arousing than positive laboratory stimuli, the differential effects of cortisol administration on angry faces may be due to either the valence or arousal qualities of these stimuli. We cannot differentiate between these factors in the present study. However, in all likelihood, valence and arousal together influence the motivational significance of these stimuli, to prepare the individual for rapid behavioral responses to stimuli that signal potential danger.

Finally, it should be noted that in order to avoid interactions with hormonal cycling in females, the findings of the present study were based on male participants only, and it remains to be tested whether similar effects emerge for females.

In conclusion, both the behavioral and ERP analyses showed that cortisol enhanced approach-avoidance congruency effects towards angry faces in high avoidant individuals only. ERP analyses showed that amplitudes of both early (P150) and late (P3) positive components were enhanced, suggesting increased processing of threat stimuli after cortisol administration. Together, these results suggest a context-specific effect of cortisol on processing of, and adaptive responses to, motivationally significant threat stimuli, particularly in participants highly sensitive to threat signals. These effects may be relevant for the study of stress and avoidance reactions in patients characterized by strong avoidance tendencies and sensitivity to social threat, such as patients with social anxiety disorder.

\section{Acknowledgements}

The authors thank Lieke Wiggers, Frans Clements and the lab assistants of the Department of Clinical Neurophysiology of the Leiden University Medical Centre (LUMC) for their assistance in data collection; Cor Kramer and Robert Reijntjes for the technical support; Hans van Pelt and Bart Ballieux for the cortisol analyses at the Clinical Chemical lab of the LUMC; Ellen de Bruijn for her advise on ERP analysis, and Bernet Elzinga for her comments on earlier versions of this paper. The work of K. Roelofs and K.R. Ridderinkhof was supported by grants from the Netherlands Organization for Scientific Research (NWO).

\section{References}

Adolphs, R., 2002. Neural systems for recognizing emotion. Current Opinion in Neurobiology 12, 169-177.

Ashley, V., Vuilleumier, P., Swick, D., 2004. Time course and specificity of event-related potentials to emotional expressions. Neuroreport 15, 211-216.

Bar, M., 2003. A cortical mechanism for triggering top-down facilitation in visual object recognition. Journal of Cognitive Neuroscience 15, 600-609.

Bar-Haim, Y., Lamy, D., Glickman, S., 2005. Attentional bias in anxiety: a behavioral and ERP study. Brain and Cognition 59, 11-22.

Bögels, S.M., Reith, W., 1999. Validity of two questionnaires to assess social fears: the Dutch Social Phobia and anxiety inventory and the blushing, trembling and sweating questionnaire. Journal of Psychopathology and Behavioral Assessment 21, 51-66.

Buss, K.A., Schumacher, J.R.M., Dolski, I., Kalin, N.H., Goldsmith, H.H., Davidson, R.J., 2003. Right frontal brain activity, cortisol, and withdrawal behavior in 6-month-old infants. Behavioral Neuroscience 117, 11-20.

Carver, C.S., White, T.L., 1994. Behavioral inhibition, behavioral activation, and affective responses to impending reward and punishment: the BIS/BAS scales. Journal of Personality and Social Psychology 67, 319-333.

Chen, M., Bargh, J.A., 1999. Consequences of automatic evaluation: immediate behavioral predispositions to approach or avoid the stimulus. Personality and Social Psychology Bulletin 25, 215-224.

Cloninger, C.R., Przybeck, T.R., Svrakic, D.M., Wetzel, R.D., 1994. The Temperament and Character Inventory (TCI): A Guide to its Development and Use. Center for Psychobiology of Personality, St. Louis.

Cools, R., Calder, A.J., Lawrence, A.D., Clark, L., Bullmore, E., Robbins, T.W., 2005. Individual differences in threat sensitivity predict serotonergic modulation of amygdala response to fearful faces. Psychopharmacology 180, 670-679.

Cuthbert, B.N., Schupp, H.T., Bradley, M.M., Birbaumer, N., Lang, P.J., 2000. Brain potentials in affective picture processing: covariation with autonomic arousal and affective report. Biological Psychology 52, 95-111.

De Kloet, E.R., Oitzl, M.S., Joëls, M., 1999. Stress and cognition: are corticosteroids good or bad guys? Trends in Neurosciences 22, 422-426.

De la Rie, S.M., Duijsens, I.J., Cloninger, C.R., 1998. Temperament, character, and personality disorders. Journal of Personality Disorders 12, 362-372.

Eimer, M., Holmes, A., 2002. An ERP study on the time course of emotional face processing. Neuroreport 13, 427-431.

Eimer, M., Holmes, A., McGlone, F.P., 2003. The role of spatial attention in the processing of facial expression: an ERP study of rapid brain responses to six basic emotions. Cognitive, Affective, and Behavioral Neuroscience $3,97-110$

Ekman, P., Friesen, W.V., 1976. Pictures of Facial Affect. Consulting Psychologist Press, Palo Alto.

Elzinga, B.M., Roelofs, K., 2005. Cortisol-induced impairments of working memory require acute sympathetic activation. Behavioral Neuroscience $119,98-103$.

Fitts, P.M., 1954. The information capacity of the human motor system in controlling the amplitude of movement. Journal of Experimental Psychology $47,381-391$.

Fox, E., Russo, R., Dutton, K., 2002. Attentional bias for threat: evidence for delayed disengagement from emotional faces. Cognition and Emotion 16, 355-379.

Goldberg, D.P., 1978. Manual of the General Health Questionnaire. NFER, Windsor. 
Gratton, G., Coles, M.G.H., Donchin, E., 1983. A new method for off-line removal of ocular artifact. Electroencephalography and Clinical Neurophysiology $55,468-484$.

Gray, J.A., 1982. The neuropsychology of anxiety: an inquiry into the functions of the septo-hippocampal system. Behavioral and Brain Sciences 5, 469-484.

Gray, J.A., McNaughton, N., 2000. The Neuropsychology of Anxiety: An Enquiry into the Functions of the Septo-Hippocampal System. University Press, Oxford.

Harmer, C.J., Thilo, K.V., Rothwell, J.C., Goodwin, G.M., 2001. Transcranial magnetic stimulation of medial-frontal cortex impairs the processing of angry facial expressions. Nature Neuroscience 4, 17-18.

Hornak, J., Bramham, J., Rolls, E.T., Morris, R.G., O’Doherty, J., Bullock, P.R., et al., 2003. Changes in emotion after circumscribed surgical lesions of the orbitofrontal and cingulate cortices. Brain 126, 1691-1712.

Huang, Y.X., Luo, Y.J., 2006. Temporal course of emotional negativity bias: an ERP study. Neuroscience Letters 398, 91-96.

Kalin, N.H., Larson, C., Shelton, S.E., Davidson, R.J., 1998a. Asymmetric frontal brain activity, cortisol, and behavior associated with fearful temperament in rhesus monkeys. Behavioral Neuroscience 112, 286-292.

Kalin, N.H., Shelton, S.E., Rickman, M., Davidson, R.J., 1998b. Individual differences in freezing and cortisol in infant and mother rhesus monkeys. Behavioral Neuroscience 112, 251-254.

Kalin, N.H., Shelton, S.E., Davidson, R.J., 2000. Cerebrospinal fluid corticotropin-releasing hormone levels are elevated in monkeys with patterns of brain activity associated with fearful temperament. Biological Psychiatry 47, 579-585.

Kawasaki, H., Adolphs, R., Kaufman, O., Damasio, H., Damasio, A.R., Granner, M., et al., 2001. Single-neuron responses to emotional visual stimuli recorded in human ventral prefrontal cortex. Nature Neuroscience 4, $15-16$.

Koeter, M.W.J., Ormel, J., 1991. General Health Questionnaire: Nederlandse bewerking-Handleiding. Swets \& Zeitlinger, Lisse, The Netherlands.

Krolak-Salmon, P., Fischer, C., Vighetto, A., Mauguière, F., 2001. Processing of facial emotional expression: spatio-temporal data as assessed by scalp event-related potentials. European Journal of Neuroscience 13, 987-994.

LeDoux, J.E., 2002. Synaptic Self: How Our Brains Become Who We Are. Viking, New York.

Lundqvist, D., Flykt, A., Öhman, A., 1998. The Karolinska Directed Emotional Faces. Karolinska Institute, Stockholm.

Markman, A.B., Brendl, C.M., 2005. Constraining theories of embodied cognition. Psychological Science 16, 6-10.

Mathews, A., Macleod, C., 1994. Cognitive approaches to emotion and emotional disorders. Annual Review of Psychology 45, 25-50.

Matsumoto, D., Ekman, P., 1988. Japanese and Caucasian Facial Expressions of Emotion (JACFEE) [slides]. University of California, Human Interaction Laboratory, San Francisco.

McClure, E.B., Monk, C.S., Nelson, E.E., Zarahn, E., Leibenluft, E., Bilder, R.M., et al., 2004. A developmental examination of gender differences in brain engagement during evaluation of threat. Biological Psychiatry 55, 1047-1055.

Meaney, M.J., Aitken, D.H., 1985. $\left[{ }^{3}\right.$ H]dexamethasone binding in rat frontal cortex. Brain Research 328, 176-180.

Mogg, K., Bradley, B.P., 2002. Selective orienting of attention to masked threat faces in social anxiety. Behaviour Research and Therapy 40, 1403-1414.

Nieuwenhuis, S., Aston-Jones, G., Cohen, J.D., 2005. Decision making, the P3, and the locus coeruleus-norepinephrine system. Psychological Bulletin $131,510-532$.

Pizzagalli, D., Regard, M., Lehmann, D., 1999. Rapid emotional face processing in the human right and left brain hemispheres: an ERP study. Neuroreport 10, 2691-2698.

Putman, P., Hermans, E.J., Van Honk, J., 2007. Exogenous cortisol shifts a motivated bias from fear to anger in spatial working memory for facial expressions. Psychoneuroendocrinology 32, 14-21.

Radley, J.J., Sisti, H.M., Hao, J., Rocher, A.B., McCall, T., Hof, P.R., et al., 2004. Chronic behavioral stress induces apical dendritic reorganization in pyramidal neurons of the medial prefrontal cortex. Neuroscience 125, 1-6.
Ridderinkhof, K.R., Ullsperger, M., Crone, E.A., Nieuwenhuis, S., 2004. The role of the medial frontal cortex in cognitive control. Science 306, 443-447.

Roelofs, K., Elzinga, B.M., Rotteveel, M., 2005. The effects of stress-induced cortisol responses on approach-avoidance behavior. Psychoneuroendocrinology 30, 665-677.

Roelofs, K., Minelli, A., Mars, R., van Peer, J., Toni, I., in preparation. On the cerebral control of social emotional behavior.

Rolls, E.T., 2000. Précis of The brain and emotion. Behavioral and Brain Sciences 23, 177-191.

Roozendaal, B., McReynolds, J.R., McGaugh, J.L., 2004. The basolateral amygdala interacts with the medial prefrontal cortex in regulating glucocorticoid effects on working memory impairment. Journal of Neuroscience 24, 1385-1392.

Rotteveel, M., Phaf, R.H., 2004. Automatic affective evaluation does not automatically predispose for arm flexion and extension. Emotion 4, 156-172.

Sapolsky, R.M., 1990. Adrenocortical function, social rank, and personality among wild baboons. Biological Psychiatry 28, 862-878.

Sapolsky, R.M., Romero, L.M., Munck, A.U., 2000. How do glucocorticoids influence stress responses? Integrating permissive, suppressive, stimulatory, and preparative actions. Endocrine Reviews 21, 55-89.

Sato, W., Kochiyama, T., Yoshikawa, S., Matsumura, M., 2001. Emotional expression boosts early visual processing of the face: ERP recording and its decomposition by independent component analysis. Neuroreport 12 , 709-714.

Schupp, H.T., Öhman, A., Junghofer, M., Weike, A.I., Stockburger, J., Hamm, A.O., 2004. The facilitated processing of threatening faces: an ERP analysis. Emotion 4, 189-200.

Smillie, L.D., Pickering, A.D., Jackson, C.J., 2006. The new reinforcement sensitivity theory: implications for personality measurement. Personality and Social Psychology Review 10, 320-335.

Solarz, A.K., 1960. Latency of instrumental responses as a function of compatibility with the meaning of eliciting verbal signs. Journal of Experimental Psychology 59, 239-245.

Spielberger, C.D., 1983. Manual for the State-Trait Anxiety Inventory (STAIform Y). Consulting Psychologists Press, Palo Alto.

Strauss, M.M., Makris, N., Aharon, I., Vangel, M.G., Goodman, J., Kennedy, D.N., et al., 2005. fMRI of sensitization to angry faces. Neuroimage 26, 389-413.

Turner, S.M., Beidel, D.C., Dancu, C.V., Stanley, M.A., 1989. An empirically derived inventory to measure social fears and anxiety: the Social Phobia and Anxiety Inventory. Psychological Assessment 1, 35-40.

Van Aken, M.O., Romijn, J.A., Miltenburg, J.A., Lentjes, E.G.W.M., 2003. Automated measurement of salivary cortisol. Clinical Chemistry 49, $1408-1409$.

Van der Ploeg, H.M., 2000. Handleiding bij de Zelf-Beoordelingsvragenlijst. Een Nederlandstalige bewerking van de Spielberger State-Trait Anxiety Inventory (2e gewijzigde druk). (Manual of the Self-Report questionnaire. A Dutch translation of the Spielberger State-Trait Anxiety Inventory), 2nd modified ed. Swets \& Zeitlinger B.V., Lisse, The Netherlands.

Van Honk, J., Tuiten, A., van den Hout, M., Koppeschaar, H., Thijssen, J., de Haan, E., et al., 1998. Baseline salivary cortisol levels and preconscious selective attention for threat: a pilot study. Psychoneuroendocrinology 23, 741-747.

Van Veen, V., Carter, C.S., 2002. The timing of action-monitoring processes in the anterior cingulate cortex. Journal of Cognitive Neuroscience 14, 593-602.

Van Veen, V., Holroyd, C.B., Cohen, J.D., Stenger, V.A., Carter, C.S., 2004. Errors without conflict: implications for performance monitoring theories of anterior cingulate cortex. Brain and Cognition 56, 267-276.

Williams, L.M., Palmer, D., Liddell, B.J., Song, L., Gordon, E., 2006. The 'when' and 'where' of perceiving signals of threat versus non-threat. NeuroImage 31, 458-467.

Wolf, O.T., 2003. HPA axis and memory. Best Practice and Research Clinical Endocrinology and Metabolism 17, 287-299.

Yeung, N., Botvinick, M.M., Cohen, J.D., 2004. The neural basis of error detection: conflict monitoring and the error-related negativity. Psychological Review 111, 931-959. 